Case Report : Open Access

\title{
Myopathy Revealing Parathyroid Carcinoma
}

\author{
KS Moalla', N Bouzidi',2, M Damak ${ }^{1,2}$, M Abid $^{2,3}$ and C Mhiri',2* \\ ${ }^{1}$ Department of Neurology, Habib Bourguiba University Hospital, Tunisia \\ ${ }^{2}$ Department of medicine, University of Sfax, Tunisia \\ ${ }^{3}$ Department of Endocrinology, Hédi Chaker University Hospital, Tunisia
}

*Corresponding author: Pr. Chokri Mhiri, Department of Neurology, Habib Bourguiba Hospital - CP 3029 Sfax, Tunisia, Tel: +216-95-83-5083, E-mail:mhiri.chokri@gmail.com

\begin{abstract}
Primary hyperparathyroidism (PHPT) is defined as an inappropriate and excessive secretion of parathyroid hormone (PTH). It is usually asymptomatic and often diagnosed after a fortuitous discovery of hypercalcemia. We report the case of severe myopathy revealing PHPT secondary to parathyroid carcinoma (PC) with lung metastasis.

A 67-year-old man presented with a 6 months history of muscle weakness, gait disturbance and difficulties to climb stairs. He was also suffering from bone pain and mentioned $20 \mathrm{Kg}$ weight loss. Physical examination disclosed myopathic syndrome with waddling gait and motor deficit predominating in pelvic girdles and proximal segments of limbs. Laboratory tests revealed severe hypercalcemia associated to a significant increase of PTH. Etiologic screening showed carcinoma of the parathyroid gland with lung metastasis.

Although myopathic syndrome exceptionally reveals PHPT, clinicians should be aware of this endocrinopathy facing an acquired myopathy in the elderly.
\end{abstract}

\section{Keywords}

Primary hyperparathyroidism, Parathyroid neoplasms, Muscular diseases

\section{Clinical Case}

A 67-year-old man, with no medical history, was referred to our department for gait disturbance. In fact, since six months, he developed progressively lower limbs heaviness with difficulties to walk and climb stairs. He did not report myalgia or dysphagia and there was no cutaneous sign. However, he was suffering from diffuse bone pain (particularly in the spine and pelvis) and anorexia with $20 \mathrm{Kg}$ weight loss. He also complained from chronic constipation and noticed change of his voice, which became slurred.
Physical examination disclosed proximal muscle deficit particularly the pelvic girdle (MRC grade 3) with waddling gait and inability to get up from the floor. A moderate muscle wasting was also observed. Deep tendon reflexes, plantar responses and sensory system were normal. Electromyography (EMG) study showed both myogenic and neurogenic features. Careful examination found an irregular solid and painless nodule in the left side of the neck without satellite lymph node hypertrophy. This cervical mass was completely ignored by the patient.

Blood tests showed a normal serum creatine kinase (CK) level (35 IU/L).The FT4 and TSH rate were normal. There

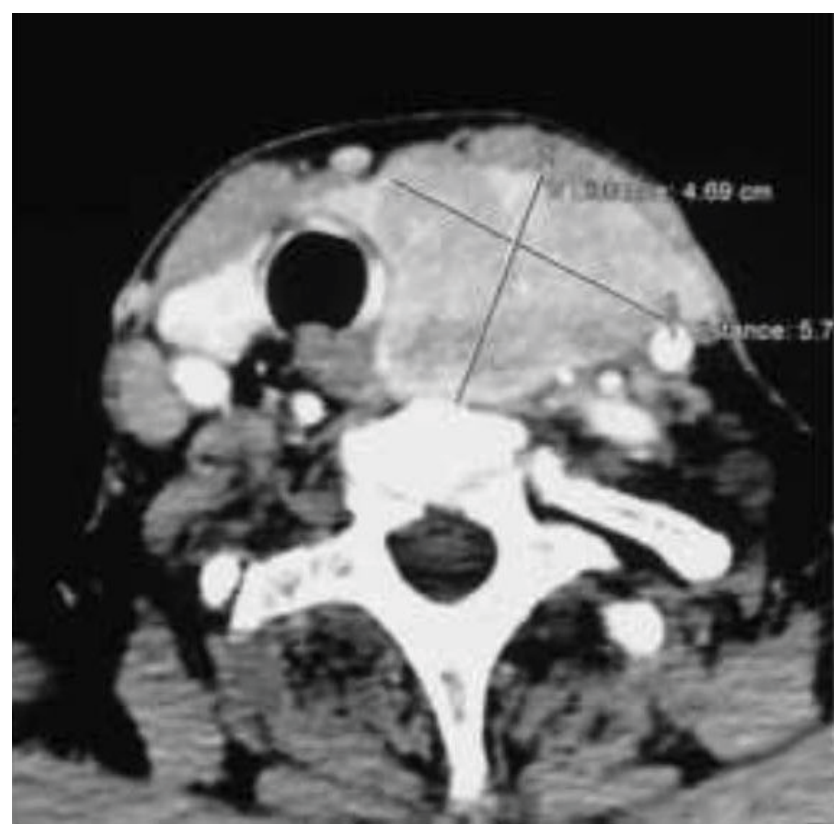

Figure 1: Computed tomography of the cervix performed after contrast injection demonstrating a left cervical mass that compresses the esophagus and the trachea.

\section{ClinMed} International Library

Citation: Moalla KS, Bouzidi N, Damak M, Abid M, Mhiri C (2017) Myopathy Revealing Parathyroid Carcinoma. Int Arch Endocrinol Clin Res 3:007

Received: August 08, 2016: Accepted: February 18, 2017: Published: February 21, 2017 Copyright: @ 2017 Moalla KS, et al. This is an open-access article distributed under the terms of the Creative Commons Attribution License, which permits unrestricted use, distribution, and reproduction in any medium, provided the original author and source are credited. 


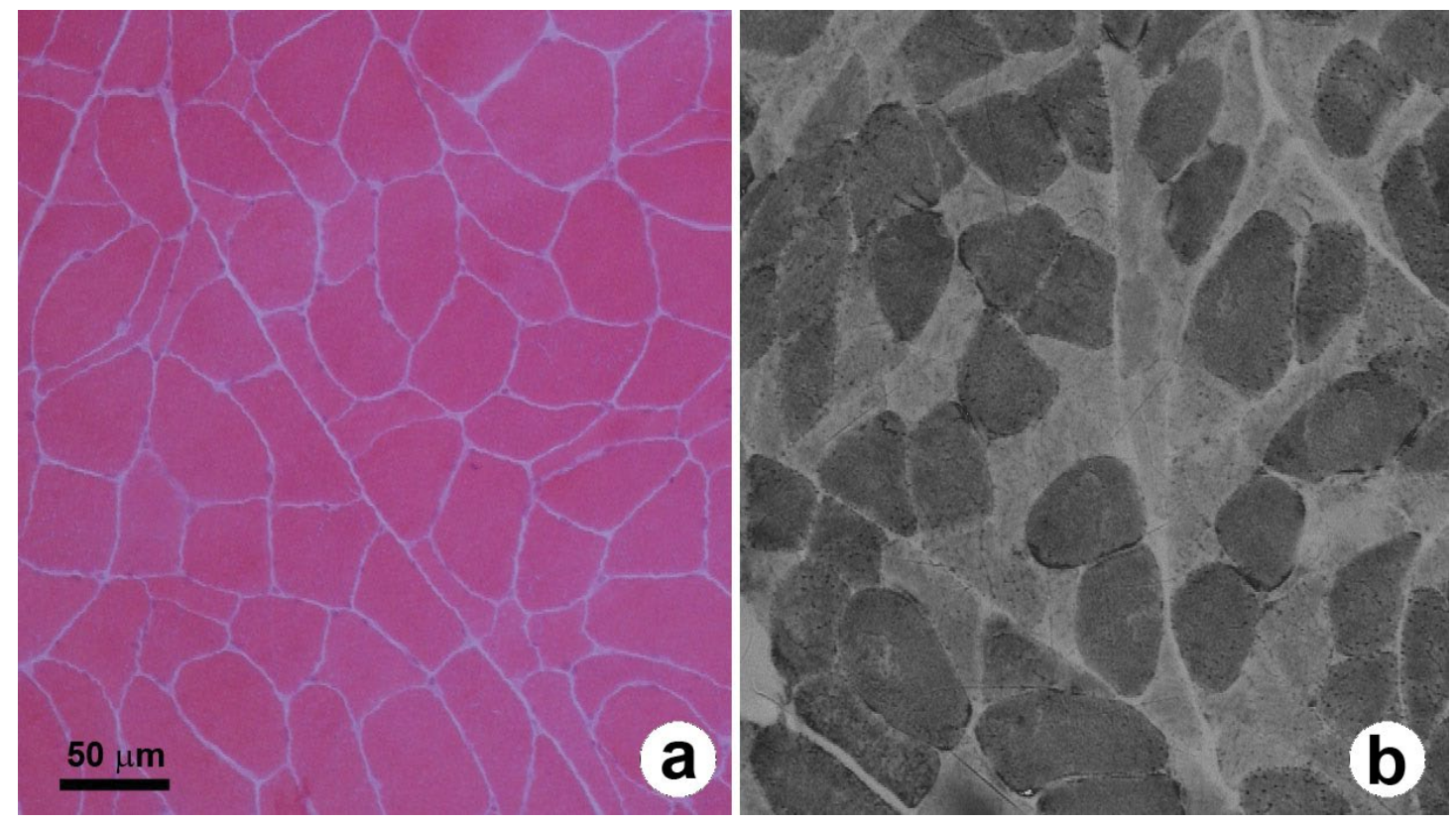

Figure 2: a) Frozen transverse section of the deltoid muscle. Hematoxylin eosin staining shows disparity of fiber size some angulated fibers; b) NADH tetrazolium reductase reaction yields that atrophy involves particularly type 2 fibers (b).

was a severe hypercalcemia $(4 \mathrm{mmol} / \mathrm{l})$ which was treated urgently as it is a potentially life threatening. Serum phosphate level was normal $(1 \mathrm{mmol} / \mathrm{l})$. Given this unbalance of serum calcium, we performed parathyroid hormone $(\mathrm{PTH})$ assay and discovered a very high level at $3951 \mathrm{pg} / \mathrm{ml}$ (normal range: 14 to $57 \mathrm{pg} / \mathrm{ml})$. Alkaline phosphatase level was also very high at $506 \mathrm{IU} / \mathrm{l}$ (normal range: 40 to $150 \mathrm{IU} / \mathrm{l})$. Serum vitamin D was at the lower limit of normal (25-OH vitamin D: $11.6 \mathrm{ng} / \mathrm{ml}$ ). There was a moderate renal impairment (creatinine clearance $44 \mathrm{ml} / \mathrm{min}$ ) and hypercalciuria (12 mmol/24 h; normal $<7.5 \mathrm{mmol} / 24 \mathrm{~h}$ ).

At ultrasound, the cervical mass measured $6 \mathrm{~cm}$, had ill-defined limits and was richly vascularized at color Doppler. CT-scan confirmed the parathyroid nodule which contained microcalcifications. This tumor involved the left thyroid lobe and repulsed the trachea to the back, the esophagus to the right and the homo lateral jugular vein and carotid artery outside (Figure 1).

Histological study of the deltoid muscle biopsy was performed on frozen and paraffin embedded sections. Hematoxylin eosin staining showed disparity of fiber size some angulated fibers (Figure 2a). NADH tetrazolium reductase reaction yielded that atrophy involved particularly type 2 fibers (Figure 2b). This selective type II atrophy is a non-specific pattern and could be observed in steroid or endocrine myopathy. There were neither necrotic fibers nor regenerative process. Immunohistochemistry showed positive staining for dystrophin, sarcoglycans and dysferlin. Serial sections of paraffin embedded sample didn't find any inflammatory infiltrate nor rimmed vacuoles.

X-ray of the pelvis, femurs, humerus and hands exhibited patchy osteolytic areas (Figure 3a). Skull radiography showed "pepper pot" appearance (Figure $3 b$ ). Bone densitometry demonstrated severe bone deminer- alization (femoral T-score at -8.3; normal range between +1 and -1 ). Abdominal pelvic ultrasound revealed multiple renal and bladder stones.

These findings suggested the invasive character and the malignant type of the tumor. Body scan revealed multiple pulmonary metastases and brown tumors in the skull and mandible. All these clinical and paraclinical data were strongly suggestive of a metastatic parathyroid carcinoma (PC) complicated by myopathy. The patient underwent surgical resection of the parathyroid mass. Pathological examination of the frozen sections confirmed the malignancy of the tumor (capsular, vascular and perineural invasions). Regarding this result, the thyroid gland and the regional lymph nodes were completely removed. During follow-up, we observed progressive global improvement and a slight amelioration of muscle strength (MRC grade 3+). Biological control showed decreased PTH level to $820 \mathrm{pg} / \mathrm{ml}$, hypocalcaemia, hypophosphatemia and increased alkaline phosphatase evoking a "Hungry Bone Syndrome" (HBS), despite calcium and vitamin $\mathrm{D}$ supplementation.

\section{Discussion}

Primary hyperparathyroidism (PHPT) is caused by increased secretion of PTH. This common endocrine disorder occurs usually in elderly, most often around the age of 60 . The estimated incidence is approximately 20.5 cases/100.000 persons per year. Women are three times more often affected than men [1]. Diagnosis of PHPT is suggested by hypercalcemia, hypophosphatemia and raised PTH in appropriate clinical settings [2]. However, it is usually asymptomatic and hypercalcemia is often found unexpectedly on biochemical screening. When symptomatic, the most frequent signs are bone and abdominal pain, constipation, nausea, vomiting and nephrolithiasis [3]. 

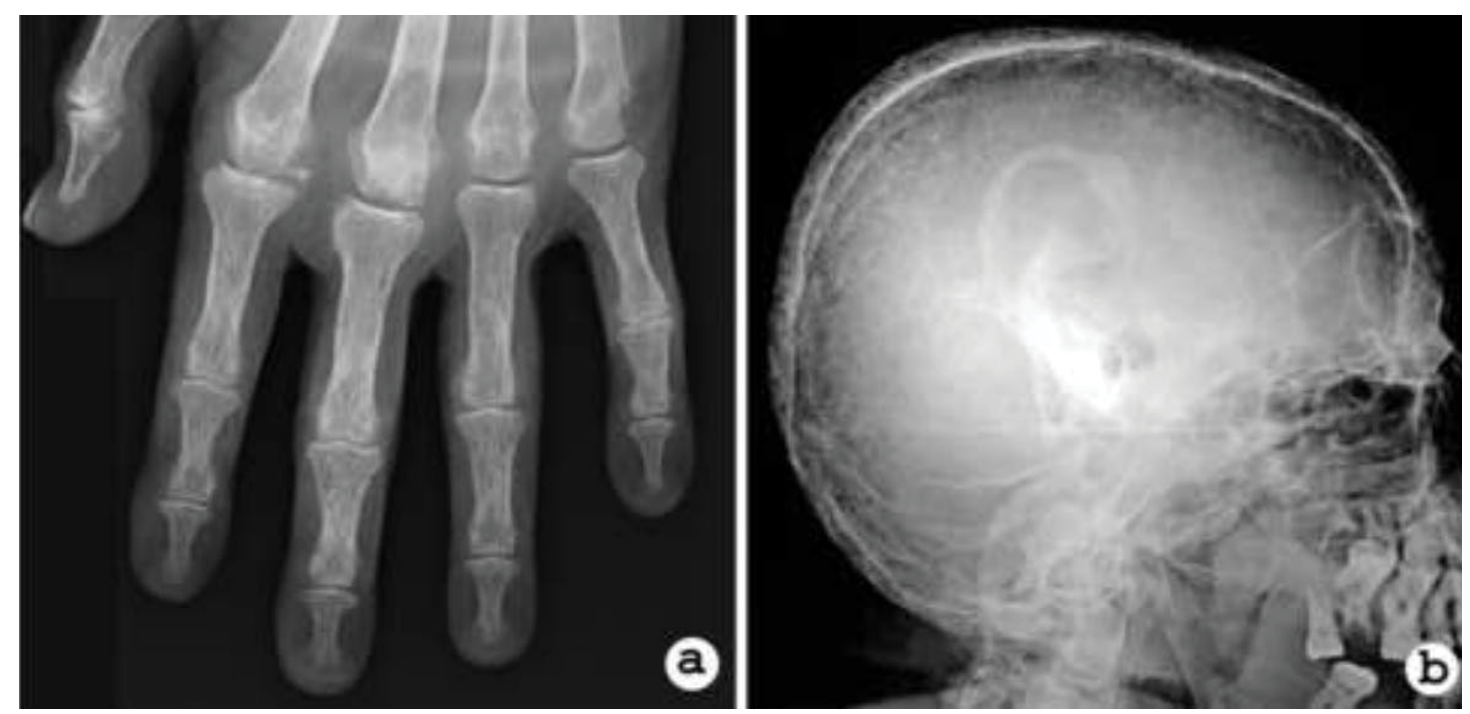

Figure 3: a) Hand X-ray revealing diffuse demineralization and erosion of phalanges; b) Radiography of skull showing a 'pepper pot' appearance.

Neurologic manifestations of PHPT are uncommon and in rare cases, they reveal the disease. Both central and peripheral nervous system can be involved. Psychiatric symptoms are frequent such as anxiety, depression, hypersomnia, impaired thinking and memory loss. In some cases, patient complains from headache, presents seizure, confusion and/or consciousness alteration. These symptoms are related to very severe hypercalcemia secondary to excessive PTH release [2]. Besides the importance of neuropsychiatric complains, neurological examination shows minimal changes such as brisk tendon reflexes. In one case report, spasticity was found in the lower limbs with Babinski sign [4]. Cramps and paresthesia are reported in approximately $50 \%$ of patients [5]. In addition, $29 \%$ to $57 \%$ of patients experience hypoesthesia with stocks-gloves distribution, diminished vibratory sense in the extremities and decreased muscle stretch reflexes suggestive of an underlying peripheral neuropathy $[5,6]$.

The myopathy associated with PHPT can rarely reveal the disease [7]. It is characterized by gradual onset of symmetric proximal weakness with atrophy and sometimes muscle pain $[8,9]$. Proximal leg weakness is responsible for waddling gait and in advanced stage the patient became bedridden. Only one case of severe neck extensor weakness, the so called "dropped head syndrome", and severe respiratory muscle involvement were reported $[10,11]$. It was reported several patients with hoarseness and dysphagia [6]. CK level is usually normal [12]. EMG usually shows normal muscle activity, some short duration motor unit potentials can be observed. The muscle biopsy specimen shows type II atrophy but there is no fiber necrosis and regeneration nor inflammatory infiltrate [6].

Proximal muscle deficit in the elderly is not specific and many differential diagnoses can be discussed especially inflammatory myopathies (polymyositis, dermatomyositis ...) by far more frequent. In our case, the absence of myalgia, the normal CK level and the absence of inflammatory infiltrates on muscle biopsy exclude these diagnoses. Toxic myopathies are also common in the elderly but our patient had not been exposed to statins, colchicine or steroids... CK level is usually increased in theses toxic myopathies. Myopathy related to disorders of thyroid metabolism may have similar clinical pattern of PHPT's myopathy. However, other signs of thyroid dysfunction are usually present and the diagnosis is confirmed with the determination of low or high thyroid hormone levels [13].

The exact physiopathology of muscular dysfunction in PHPT is not well known. It is established that hypercalcemia induces a reduction of neuromuscular excitability and can cause muscle weakness, however, fatigue and muscle weakness are much more common in PHPT. Therefore, some authors believe that myopathic syndrome is more related to the increase of PTH than hypercalcemia [14]. Other authors found that there is a thickening of the arterioles and the basement membrane in the smallest endomysial vessels suggesting that vascular factors may have a significant role in the pathogenesis of the myopathy in PHPT $[7,15]$. In addition, osteomalacia secondary to vitamin $\mathrm{D}$ deficiency may aggravate muscle dysfunction [16]. Furthermore, vitamin D status appears inversely correlated with PTH levels, with several studies suggesting increased severity of PHPT in vitamin D-deficient individuals as well as a higher risk of severe postoperative HBS $[17,18]$.

About $80 \%$ of cases of PHPT are related to benign tumor (adenoma) developed in one of the parathyroid glands. In other cases, hyperactivity of the parathyroid is secondary to multiple tumors of the glands or hyperplasia. Rarely, PHPT is caused by parathyroid carcinoma (PC) $[1,2]$. It is a malignant neoplasm that represents less than $0.005 \%$ of all cancers $[19,20]$. If we consider all patients suffering from PHPT, PC represents 0.5 to $5 \%$ of cases [21]. It occurs with equal frequency in men and women. Classically, the disease is described to occur one decade earlier than parathyroid adenoma (mean age 50 years) [22]. To our knowledge this is the first case of $\mathrm{PC}$ revealed by a myopathic syndrome.

This cancer causes a challenge for diagnosis and treatment because of its rarity, overlapping features with be- 
nign parathyroid disease, and lack of distinct characteristics. Certain conditions are considered to be associated to a higher risk of parathyroid carcinoma, without being specific. Severe hypercalcemia, young age, male gender, hypercalcemic crisis, concomitant renal and bone involvement, as well as the presence of a palpable neck mass (more than $3 \mathrm{~cm}$ ) should raise the suspicion of parathyroid carcinoma. Hoarseness of voice, as a sign of recurrent laryngeal nerve palsy due to local invasion, is highly suggestive of malignancy, since it is very uncommon in parathyroid adenoma. Approximately one third of patients have metastatic lesions at presentation like our patient. The most common sites of metastasis are lungs, followed by liver and bones $[20,23]$. Early surgical resection of the primary tumor with clear margins remains the best curative treatment [24]. Successful surgery is followed by a prompt normalization of the PTH level, in consequence muscle strength improves and myopathic syndrome regresses gradually [25]. Clinical follow-up and monitoring of serum calcium and PTH levels are mandatory. Local recurrence is frequent (in one third of the patients) and the 5-year survival is up to 40 $86 \%$ [26]. Surgical treatment of recurrence and metastasis is recommended where feasible and compatible with the patient's general condition to alleviate clinical symptoms and normalize temporarily laboratory abnormalities. The efficacy of classical adjuvant therapies, such as radiotherapy and chemotherapy, in management of persistent, recurrent, or metastatic disease is controversial. In metastatic PC, the goal of therapeutic support is to control the PTH-driven hypercalcemia that represents the primary cause of death. In the case of metastatic disease and when surgery is no longer indicated, combined techniques, such as powerful bisphosphonates, new anti-resorbtive agents, calcimimetics, radiofrequency ablation and immunotherapy, are useful tools in controlling the complications of severe hypercalcemia [27].

\section{References}

1. Wermers RA, Khosla S, Atkinson EJ, Achenbach SJ, Oberg AL, et al. (2006) Incidence of primary hyperparathyroidism in Rochester, Minnesota, 1993-2001: an update on the changing epidemiology of the disease. $J$ Bone Miner Res 21: 171-177.

2. Blanchard C, Mirallié E, Mathonnet M (2010) Hyperparathyroïdie primaire sporadique. J Chir Viscérale 147: 360-370.

3. Cormier C (2012) Hyperparathyroïdie primitive. Rev Rhum Monogr 79: 233-238.

4. Carnevale V, Minisola S, Romagnoli E, D'Erasmo E, Bragoni M, et al. (1992) Concurrent improvement of neuromuscular and skeletal involvement following surgery for primary hyperparathyroidism. J Neurol 239: 57.

5. Turken SA, Cafferty M, Silverberg SJ, De La Cruz L, Cimino C, et al. (1989) Neuromuscular involvement in mild, asymptomatic primary hyperparathyroidism. Am J Med 87: 553-557.

6. Patten BM, Bilezikian JP, Mallette LE, Prince A, Engel WK, et al. (1974) Neuromuscular disease in primary hyperparathyroidism. Ann Intern Med 80: 182-193.

7. Cholod EJ, Haust MD, Hudson AJ, Lewis FN (1970) Myopathy in primary familial hyperparathyroidism. Clinical and morphologic studies. Am J Med 48: 700-707.
8. Lafferty FW (1981) Primary hyperparathyroidism. Changing clinical spectrum, prevalence of hypertension, and discriminant analysis of laboratory tests. Arch Intern Med 141: 1761-1766.

9. Muthukrishnan J, Jha S, Modi KD, Jha R, Kumar J, et al. (2008) Symptomatic primary hyperparathyroidism: a retrospective analysis of fifty one cases from a single centre. J Assoc Physicians India 56: 503-507.

10. Rymanowski JV, Twydell PT (2009) Treatable dropped head syndrome in hyperparathyroidism. Muscle Nerve 39: 409-410.

11. Gentric A, Pennec YL (1994) Fatal primary hyperparathyroidism with myopathy involving respiratory muscles in an old woman. J Am Geriatr Soc 42: 1306.

12. Berthelot J-M (2008) Pathologies endocriniennes et muscles. Rev Rhum 75: 172-177.

13. Cherin $P$ (2014) Les myopathies du sujet âgé. NPG Neurol-Psychiatr-Gériatrie 14: 4-10.

14. Ubogu EE, Ruff RL, Kaminski HJ (2004) Endocrine myopathies. Myol, McGraw-Hill, NY, 1713-1738.

15. ENGEL MB (1952) Mobilization of mucoprotein by parathyroid extract. AMA Arch Pathol 53: 339-351.

16. Moulias S (2004) Myopathies du sujet âgé. Rev Rhum 71: 510-514.

17. Garingarao CJ, Paz-Pacheco E, Jimeno CA (2014) Primary hyperparathyroidism from a probable ectopic parathyroid adenoma with severe skeletal disease and vitamin $D$ deficiency. BMJ Case Rep 2014.

18. Rastogi A, Bhadada SK, Bhansali A (2013) Pseudoarthrosis and fracture: interaction between severe vitamin $D$ deficiency and primary hyperparathyroidism. Singapore Med J 54: e224.

19. Dudney WC, Bodenner D, Stack BC Jr (2010) Parathyroid carcinoma. Otolaryngol Clin North Am 43: 441-453.

20. Hundahl SA, Fleming ID, Fremgen AM, Menck HR (1999) Two hundred eighty-six cases of parathyroid carcinoma treated in the U.S. between 1985-1995: a National Cancer Data Base Report. The American College of Surgeons Commission on Cancer and the American Cancer Society. Cancer 86: 538-544.

21. Schoretsanitis G, Daskalakis M, Melissas J, Tsiftsis DD (2009) Parathyroid carcinoma: clinical presentation and management. Am J Otolaryngol 30: 277-280.

22. Al-Kurd A, Mekel M, Mazeh H (2014) Parathyroid carcinoma. Surg Oncol 23: 107-114.

23. Wei CH, Harari A (2012) Parathyroid carcinoma: update and guidelines for management. Curr Treat Options Oncol 13: 11-23.

24. Mucci-Hennekinne S, Desolneux G, Luyckx F, Gibelin H, Mirallié E, et al. (2008) Parathyroid carcinoma: multicenter study of 17 patients. J Chir (Paris) 145: 133-137.

25. Horak HA, Pourmand R (2000) Endocrine myopathies. Neurol Clin 18: 203-213.

26. Shane E (2001) Clinical review 122: Parathyroid carcinoma. J Clin Endocrinol Metab 86: 485-493.

27. Betea D, Potorac I, Beckers A (2015) Parathyroid carcinoma: Challenges in diagnosis and treatment. Ann Endocrinol (Paris) 76: 169-177. 